VOL. $68(2003) \quad[45-56]$

\title{
A LOCAL APPROACH TO A CLASS OF LOCALLY FINITE GROUPS
}

\author{
A. Ballester-Bolinches and Tatiana Pedraza
}

This paper is devoted to the study of a class of generalised $p$-nilpotent groups in the universe $c \overline{\mathcal{L}}$ of all radical locally finite groups satisfying min- $q$ for every prime $q$. Some results of finite groups are extended and a characterisation of the injectors associated with this class is given.

\section{INTRODUCTION}

A property of groups is said to be "local" if it is generalised in a form referring to a prime. An interesting problem in this context is to find out whether the original property can be described as the conjunction of all the local properties for all primes. For instance, if we consider the property of finite groups of being nilpotent, a local version is that of being $p$-nilpotent, $p$ a prime. Obviously every finite nilpotent group is $p$-nilpotent and a finite group which is $p$-nilpotent for all primes $p$ is nilpotent. A similar approach to the class of locally nilpotent groups has been obtained by using the same definition of $p$-nilpotent groups as in the finite case (see [6, (1.3.5)]).

In this paper we consider another generalisation of the class of nilpotent groups. Denote by $c \overline{\mathcal{L}}$ the class of all radical locally finite groups with min- $q$ for all primes $q$ and let $\mathcal{B}$ be the class of all $c \overline{\mathcal{L}}$-groups in which every proper subgroup has a proper normal closure. This is a class of generalised nilpotent groups. It is shown in [3] and [4] that this class behaves in the universe $c \overline{\mathcal{L}}$ as the nilpotent groups in the finite universe.

Our purpose here is to introduce and study a local version of the class $\mathcal{B}$. In particular, some properties appear relating the $p$-Fitting subgroup and a new characteristic subgroup which is defined by intersections of certain types of major subgroups. This subgroup can be considered as the local version of the Frattini-like subgroup introduced by Tomkinson in [12]. Moreover, we study the injectors associated with this class in our universe.

Received 4th November, 2002

This research is supported by Proyecto BFM-2001-1667-C03-03 (MCyT) and FEDER (European Union).

Copyright Clearance Centre, Inc. Serial-fee code: 0004-9727/03 \$A2.00+0.00. 


\section{Preliminary Results}

We begin by recalling some definitions. Notation that is not specifically cited here is consistent with that used in $[6,7,10]$.

Let $U$ be a subgroup of a group $G$ and consider the properly ascending chains

$$
U=U_{0}<U_{1}<\ldots<U_{\alpha}=G
$$

from $U$ to $G$. We define $m(U)$ to be the least upper bound of the types $\alpha$ of all such chains. Clearly $m(U)=1$ if and only if $U$ is a maximal subgroup of $G$. A proper subgroup $M$ of $G$ is said to be a major subgroup of $G$ if $m(U)=m(M)$ whenever $M \leqslant U<G$. Then we define $\mu(G)$ to be the intersection of all major subgroups of $G$.

Following [14], a group $G$ is semiprimitive if it is the split extension, $G=[D] M$, of a divisibly irreducible $\mathbb{Z} M$-module $D$ by a finite soluble group $M$ with trivial core.

In the sequel all groups considered belong to the class $c \overline{\mathcal{L}}$ of all radical locally finite groups with min-q for all primes $q$.

As an attempt to extend the concept of a chief factor of a finite group we introduced the following concept. Let $G$ be a group and consider $H$ and $K$, two normal subgroups of $G$ such that $K$ is contained in $H$. Then $H / K$ is called a $\delta$-chief factor of $G$ if $H / K$ is either a minimal normal subgroup of $G / K$ or a divisibly irreducible $\mathbb{Z} G$-module, that is, $H / K$ has no proper infinite $G$-invariant subgroups.

Let $G$ be a group and consider a major subgroup $M$ of $G$. Write $M_{G}=\operatorname{Core}_{G}(M)$. Then, applying [1, Theorem 1], the factor group $G / M_{G}$ is either a finite soluble primitive group, if $M$ is a maximal subgroup of $G$, or a semiprimitive group, and therefore a Chernikov group, if $M$ is not maximal in $G$. This result motivates the following definition:

Definition: ([4]) Let $G$ be a group and let $M$ be a major subgroup of $G$. We define

$$
D_{M} / M_{G}= \begin{cases}\operatorname{Soc}\left(G / M_{G}\right), & \text { if } M \text { is a maximal subgroup of } G \\ \left(G / M_{G}\right)^{0}, & \text { if } M \text { is not a maximal subgroup of } G\end{cases}
$$

In both cases, we have that $D_{M} / M_{G}=F\left(G / M_{G}\right),\left(D_{M} / M_{G}\right) \cap\left(M / M_{G}\right)=1$ and $\mathrm{C}_{G / M_{G}}\left(D_{M} / M_{G}\right)=D_{M} / M_{G}$ for every major subgroup $M$ of $G$.

For the sake of completeness, we list some results which are needed to prove our theorems. We begin with a result that uses the Frattini-like subgroup introduced by Tomkinson to obtain a complete characterisation of the class $\mathcal{B}$ of generalised nilpotent groups analogous to the finite one for nilpotent groups and the Frattini subgroup.

ThEOREM A. ([3, Theorem 1].) Let $G$ be a group. The following statements are equivalent:

(i) $G$ is a $\mathcal{B}$-group.

(ii) $G / \mu(G)$ is a $\mathcal{B}$-group.

(iii) $G^{\prime} \leqslant \mu(G)$. 
(iv) Every major subgroup of $G$ is a normal subgroup of $G$.

(v) $G$ is a direct product of nilpotent Sylow subgroups.

(vi) $G$ is locally nilpotent and the radicable part of $G$ is central.

(vii) Every $\delta$-chief factor of $G$ is central.

Theorem B. ([3, Theorem 5].) Every group $G$ has a unique largest normal $B$ subgroup, denoted by $\delta(G)$. In fact, $\delta(G)=F(G)$, the Fitting subgroup of $G$.

Theorem C. ([3, Theorem 7].) Suppose that $G$ is a group. Then $F(G)$ is the intersection of the centralisers of all $\delta$-chief factors of $G$.

TheOREM D. ([2, Theorem 5].) Let $G$ be a group and let $H$ be a subgroup of $G$. Then $H$ is a descendant $\mathcal{B}$-subgroup of $G$ if and only if $H$ is contained in $F(G)$.

\section{The SUbGRoup $\mu_{p}(G)$ AND the Class $\mathcal{B}_{p}$}

The purpose of this section is to introduce and study a local version of the class $\mathcal{B}$. Groups in this class are called $\mathcal{B}_{p}$-groups. They play the same role in the universe $c \overline{\mathcal{L}}$ as finite $p$-nilpotent groups do in the finite one. We shall prove that this class can be associated in a natural way with a local version of Tomkinson's Frattini-like subgroup. We begin with the following definition.

Definition 1: Let $p$ be a prime. We say that a group $G$ is a $\mathcal{B}_{p}$-group if $G$ is $p$-nilpotent and the Sylow $p$-subgroups of $G$ are nilpotent.

It is clear that a finite group is a $\mathcal{B}_{p}$-group if and only if it is $p$-nilpotent. Moreover, applying Theorem A and [6, (1.3.5)], a group $G$ is in the class $\mathcal{B}$ if and only if $G$ is a $\mathcal{B}_{p}$-group for every prime $p$.

The next results analyse the behaviour of $\mathcal{B}_{p}$ as a class of groups.

Recall that a class $\mathcal{F}$ of $c \overline{\mathcal{L}}$-groups is said to be a $c \overline{\mathcal{L}}$-formation if $\mathcal{F}$ is Q-closed and it satisfies that every $c \overline{\mathcal{L}}$-group $G$ containing normal subgroups $\left\{N_{i}\right\}_{i \in I}$ such that $\bigcap_{i \in I} N_{i}=1$ and $G / N_{i} \in \mathcal{F}$ for every $i \in I$ also belongs to $\mathcal{F}$.

Taking into account the fact that the class of all $p$-nilpotent groups is a subgroupclosed $c \overline{\mathcal{L}}$-formation and using the same arguments as those used in [3, Theorem 3$]$, we have:

THEOREM 1. $\mathcal{B}_{p}$ is a subgroup-closed $c \overline{\mathcal{L}}$-formation for every prime $p$.

THEOREM 2. Let $G$ be a group. Assume that $H$ and $K$ are two normal $\mathcal{B}_{p^{-}}$ subgroups. Then $H K$ is a $\mathcal{B}_{p}$-group.

Proof: Since $H$ and $K$ are $p$-nilpotent normal subgroups, it follows that $H K$ is $p$-nilpotent. Let $P$ be a Sylow $p$-subgroup of $H K$. By $[6,(3.1 .6)]$ and $[6,(5.1 .9)]$ we have that $P=(P \cap H)(P \cap K)$ and $P \cap H$ and $P \cap K$ are Sylow $p$-subgroups of $H$ and $K$, respectively. Since $H$ and $K$ are $\mathcal{B}_{p}$-groups, we have that $P \cap H$ and $P \cap K$ are normal 
nilpotent subgroups of $P$. Consequently, we conclude that $P$ is nilpotent by Fitting's Theorem $([10,(5.2 .8)])$.

THEOREM 3. Every group $G$ has a unique largest normal $\mathcal{B}_{p}$-subgroup denoted by $\delta_{p^{\prime} p}(G)$ and called the $\mathcal{B}_{p^{\prime}}$-radical of $G$. Moreover, $F(G)=\bigcap_{p} \delta_{p^{\prime} p}(G)$.

Proof: We argue as in [3, Lemma 3]. It is easy to prove that if $G$ is a Chernikov group which is the union of a totally ordered set of normal $\mathcal{B}_{p}$-subgroups then $G$ is a $\mathcal{B}_{p}$-group. Consequently, using this fact and Theorem 1, we apply Zorn's Lemma to construct a largest normal $\mathcal{B}_{p^{-}}$-subgroup of $G, \delta_{p^{\prime} p}(G)$.

Finally the Fitting subgroup of $G$ can be described as the intersection of the $\mathcal{B}_{p}$ radicals because $F(G)$ is the largest normal $\mathcal{B}$-subgroup of $G$.

It is known that in a finite group $G$, the $p$-nilpotent radical $O_{p^{\prime} p}(G)$ is the intersection of the centralisers of all $p$-chief factors of $G$. This result has been generalised in $[6,(6.2 .4)]$ to periodic locally soluble groups. We obtain an analogous result in $c \overline{\mathcal{L}}$-groups connecting the subgroup $\delta_{p^{\prime} p}(G)$ and the $\delta$-chief factors of $G$ which are $p$-groups.

THEOREM 4. Suppose that $G$ is a group. Then $\delta_{p^{\prime} p}(G)$ is the intersection of the centralisers of all $\delta$-chief factors of $G$ which are p-groups.

Proof: The proof runs parallel to [3, Theorem 7].

Taking into account the fact that the Fitting subgroup of $G$ is the intersection of the members of the set $\left\{D_{M}: M\right.$ is a major subgroup of $\left.G\right\}$ ([4, Theorem 1]), the following result seems to be natural.

THEOREM 5. Let $G$ be a group and let $p$ be a prime. Then

$$
\delta_{p^{\prime} p}(G)=\cap\left\{D_{M}: D_{M} / M_{G} \text { is a } p \text {-group }\right\} .
$$

Proof: See the proof of $[4$, Theorem 1].

In [9] Lafuente introduces a new characteristic subgroup $\phi_{p}(G)$ (where $p$ is a prime) of a finite group $G$, satisfying $\phi(G)=\bigcap_{p} \phi_{p}(G)$. Using this subgroup he obtains some results concerning maximal subgroups, Frattini and Fitting subgroups. Following this line of thought, we extend this concept to the universe $c \overline{\mathcal{L}}$. A local version of Tomkinson's subgroup appears.

Definition 2: Let $p$ be a prime and let $G$ be a group. Denote by $\mu_{p}(G)$ the intersection of all major subgroups, $M$, of $G$ such that $D_{M} / M_{G}$ is a $p$-group, if such $M$ exists; otherwise set $\mu_{p}(G)=G$.

Obviously, we deduce from the definition that $\mu_{p}(G)$ is a characteristic subgroup of $G$ for every prime $p$ and $\mu(G)=\bigcap_{p} \mu_{p}(G)$.

Note that if $S$ and $T$ are major subgroups of $G$ such that $S_{G}=T_{G}$ then $D_{S}=D_{T}$. Consequently $\mu_{p}(G) \leqslant \delta_{p^{\prime} p}(G)$ by Theorem 5 and then $\mu_{p}(G)$ is a $\mathcal{B}_{p}$-group. 
In [9] it is proved that if $G$ is a finite group then $O_{p^{\prime} p}\left(G / \phi_{p}(G)\right)=O_{p^{\prime} p}(G) / \phi_{p}(G)$ for some prime $p$. The following result and its corollary are the $c \overline{\mathcal{L}}$-version.

PROPOSITION 1. Let $T$ be a normal subgroup of a group $G$ containing $\mu_{p}(G)$. If $T / \mu_{p}(G)$ is p-nilpotent, then $T$ is p-nilpotent.

Proof: Let $T_{p^{\prime}}$ be a Sylow $p^{\prime}$-subgroup of $T$. Then $T_{p^{\prime}}$ is a Sylow $p^{\prime}$-subgroup of $T_{p^{\prime}} \mu_{p}(G)$. Since $T / \mu_{p}(G)$ is $p$-nilpotent, it follows that $T_{p^{\prime}} \mu_{p}(G)$ is a normal subgroup of $G$. Moreover the Sylow $p^{\prime}$-subgroups of $T_{p^{\prime}} \mu_{p}(G)$ are conjugate. This implies that $G=\mathrm{N}_{G}\left(T_{p^{\prime}}\right) \mu_{p}(G)$. Assume that $\mathrm{N}_{G}\left(T_{p^{\prime}}\right)$ is a proper subgroup of $G$. Then there exists a major subgroup $M$ of $G$ such that $\mathrm{N}_{G}\left(T_{p^{\prime}}\right) \leqslant M$. Since $M$ is a proper subgroup of $G$, it follows that $\mu_{p}(G)$ is not contained in $M$ and, therefore, $D_{M} / M_{G}$ is a $p^{\prime}$-group. Assume that $\left(D_{M} / M_{G}\right) \cap\left(T M_{G} / M_{G}\right)=1$. Then $T M_{G} / M_{G} \leqslant \mathrm{C}_{G / M_{G}}\left(D_{M} / M_{G}\right)=D_{M} / M_{G}$ and hence $T$ is contained in $M_{G}$, a contradiction. Consequently, $\left(D_{M} / M_{G}\right) \cap\left(T M_{G} / M_{G}\right)$ is a non-trivial normal $p^{\prime}$-subgroup of $G / M_{G}$ contained in $T M_{G} / M_{G}$. Hence $\left(D_{M} / M_{G}\right)$ - $\cap\left(T M_{G} / M_{G}\right) \leqslant T_{p^{\prime}} M_{G} / M_{G} \leqslant M / M_{G}$ and so $\left(D_{M} / M_{G}\right) \cap\left(T M_{G} / M_{G}\right)$ is contained in $\left(M / M_{G}\right) \cap\left(D_{M} / M_{G}\right)=1$, a contradiction. Therefore $\mathrm{N}_{G}\left(T_{p^{\prime}}\right)=G$. Therefore $T_{p^{\prime}}$. is a normal subgroup of $T$ and $T$ is $p$-nilpotent.

COROLlary 1. Let $N$ be a normal subgroup of a group $G$. Then $N$ is p-nilpotent if and only if $N / N \cap \mu_{p}(G)$ is p-nilpotent. In particular, we have that $O_{p^{\prime} p}\left(G / \mu_{p}(G)\right)$ $=O_{p^{\prime} p}(G) / \mu_{p}(G)$.

Our next objective is to get a similar result replacing $O_{p^{\prime} p}(G)$ by $\delta_{p^{\prime} p}(G)$. The following extension of $[9,(1.4)]$ turns out to be crucial.

LEMMA 1. Let $G$ be a group and let $p$ be a prime. Then $\mu_{p}(G) / O_{p^{\prime}}(G)$ $=\mu\left(G / O_{p^{\prime}}(G)\right)$. Therefore $\mu_{p}(G) / O_{p^{\prime}}(G)$ is a finite $p$-group.

Proof: Suppose first that $\mu_{p}(G)=G$. We show that $G$ is a $p^{\prime}$-group. Suppose that $O_{p^{\prime}}(G)$ is a proper subgroup of $G$. Let $M$ be a major subgroup of $G$ containing $O_{p^{\prime}}(G)$. On the other hand, $G / O_{p^{\prime}}(G)$ is a $p$-group because $G=O_{p^{\prime} p}(G)$. Consequently, $G / M_{G}$ is a $p$-group. Then $D_{M} / M_{G}$ is a $p$-group, a contradiction. We conclude that $O_{p^{\prime}}(G)=G$ and the result is true in this case.

Assume now that $\mu_{p}(G)$ is a proper subgroup of $G$. We begin by proving that $O_{p^{\prime}}(G) \leqslant \mu_{p}(G)$. Since $\mu_{p}(G) \neq G$, there exists a major subgroup $M$ of $G$ such that $D_{M} / M_{G}$ is a $p$-group. Suppose that $O_{p^{\prime}}(G)$ is not contained in $M$. Then $O_{p^{\prime}}(G) M_{G} / M_{G}$ is a normal $p^{\prime}$-subgroup of $G / M_{G}$ contained in $\mathrm{C}_{G / M_{G}}\left(D_{M} / M_{G}\right)=D_{M} / M_{G}$ which is a $p$-group, a contradiction. Hence $O_{p^{\prime}}(G) \leqslant M$. Let $M / O_{p^{\prime}}(G)$ be a major subgroup of $G / O_{p^{\prime}}(G)$. Suppose that $\mu_{p}(G)$ is not contained in $M$. Then $D_{M} / M_{G}$ is a $p^{\prime}$-group. Since $\mu_{p}(G) \leqslant O_{p^{\prime} p}(G)$, we have that $\mu_{p}(G) / O_{p^{\prime}}(G)$ is a $p$-group and so $\mu_{p}(G) M_{G} / M_{G}$ is a non-trivial $p$-group. It follows that $\mu_{p}(G) M_{G} / M_{G} \leqslant \mathrm{C}_{G / M_{G}}\left(D_{M} / M_{G}\right)=D_{M} / M_{G}$, a contradiction. Therefore $\mu_{p}(G)$ is contained in $M$ and we conclude that $\mu_{p}(G) / O_{p^{\prime}}(G)$ $\leqslant \mu\left(G / O_{p^{\prime}}(G)\right)$. Let $T / O_{p^{\prime}}(G)=\mu\left(G / O_{p^{\prime}}(G)\right)$. We prove that $T \leqslant \mu_{p}(G)$. Let $M$ be 
a major subgroup of $G$ such that $D_{M} / M_{G}$ is a p-group. Then $O_{p^{\prime}}(G) \leqslant M$. Therefore $M / O_{p^{\prime}}(G)$ is a major subgroup of $G / O_{p^{\prime}}(G)$ and so $T / O_{p^{\prime}}(G) \leqslant M / O_{p^{\prime}}(G)$. We conclude that $T$ is contained in $\mu_{p}(G)$.

Applying [6, (2.5.13)] we have that $G / O_{p^{\prime}}(G)$ is a Chernikov group. Therefore $\mu_{p}(G) / O_{p^{\prime}}(G)=\mu\left(G / O_{p^{\prime}}(G)\right)$ is finite by $[\mathbf{1 3},(1.2)]$. Since $\mu_{p}(G)$ is $p$-nilpotent, we have that $\mu_{p}(G) / O_{p^{\prime}}(G)$ is a finite $p$-group.

ThEOREM 6 . Let $G$ be a group and let $p$ be a prime. Then:

(i) $\mu_{p}(G)$ is contained in $\delta_{p^{\prime} p}(G)$.

(ii) $\delta_{p^{\prime} p}\left(G / \mu_{p}(G)\right)=\delta_{p^{\prime} p}(G) / \mu_{p}(G)=F\left(G / \mu_{p}(G)\right)$ is Abelian.

(iii) $C_{G}\left(\delta_{p^{\prime} p}(G) / \mu_{p}(G)\right)=\delta_{p^{\prime} p}(G)$.

ProOF. (i) It has been already noted above.

(ii) Let $T / \mu_{p}(G)=\delta_{p^{\prime} p}\left(G / \mu_{p}(G)\right)$. Applying Theorem 4 to $G / \mu_{p}(G)$ we deduce that $T$ centralises every $\delta$-chief factor $H / K$ of $G$ such that $H / K$ is a $p$-group and $\mu_{p}(G)$. $\leqslant K \leqslant H$. Note that $D_{M} / M_{G}$ is a $\delta$-chief factor of $G$ for every major subgroup $M$ of $G$. Moreover, if $D_{M} / M_{G}$ is a $p$-group we have that $\mu_{p}(G) \leqslant M_{G}$. Consequently, $T$ $\leqslant \mathrm{C}_{G}\left(D_{M} / M_{G}\right)=D_{M}$ for every major subgroup $M$ of $G$ such that $D_{M} / M_{G}$ is a $p$ group. By Theorem 5 we conclude that $T \leqslant \delta_{p^{\prime} p}(G)$. Since the other inclusion is obvious, it follows that $\delta_{p^{\prime} p}\left(G / \mu_{p}(G)\right)=\delta_{p^{\prime} p}(G) / \mu_{p}(G)$. On the other hand, it is clear that $F\left(G / \mu_{p}(G)\right)$ is contained in $\delta_{p^{\prime} p}(G) / \mu_{p}(G)$. We prove now that $\delta_{p^{\prime} p}(G) / \mu_{p}(G)$ is Abelian. We may assume that $\mu_{p}(G) \neq G$. Let $M$ be a major subgroup of $G$ such that $D_{M} / M_{G}$ is a $p$-group. Applying Theorem 5 , it follows that $\delta_{p^{\prime} p}(G) / M_{G} \leqslant D_{M} / M_{G}$, which is Abelian. Since $\mu_{p}(G)=\cap\left\{M_{G}: D_{M} / M_{G}\right.$ is a $p$-group $\}$, we conclude that $\delta_{p^{\prime} p}(G) / \mu_{p}(G)$ is Abelian. This implies that $\delta_{p^{\prime} p}(G) / \mu_{p}(G)=F\left(G / \mu_{p}(G)\right)$.

(iii) Since every group is hyperabelian, it follows that $\mathrm{C}_{G / \mu_{p}(G)}\left(F\left(G / \mu_{p}(G)\right)\right)$ is contained in $F\left(G / \mu_{p}(G)\right)$ by $[11,(2.17)]$. By (ii) we know that $\delta_{p^{\prime} p}\left(G / \mu_{p}(G)\right)$ is Abelian. Therefore $\mathrm{C}_{G / \mu_{p}(G)}\left(\delta_{p^{\prime} p}\left(G / \mu_{p}(G)\right)\right)=\delta_{p^{\prime} p}\left(G / \mu_{p}(G)\right)$. This means that $\mathrm{C}_{G}\left(\delta_{p^{\prime} p}(G) / \mu_{p}(G)\right)=\delta_{p^{\prime} p}(G)$.

Corollary 2. Let $N$ be a normal subgroup of a group $G$. Then $N$ is a $\mathcal{B}_{p}$-group if and only if $N / N \cap \mu_{p}(G)$ is a $\mathcal{B}_{p}$-group.

Corollary 3. Let $G$ be a group and let $p$ be a prime. Then $C_{G}\left(\delta_{p^{\prime}}(G)\right)$ $\leqslant \delta_{p^{\prime} p}(G)$.

CoRollary 4. Let $G$ be a group. Then $\delta_{p^{\prime} p}\left(G / O_{p^{\prime}}(G)\right)=\delta_{p^{\prime} p}(G) / O_{p^{\prime}}(G)$ for every prime $p$.

Proof: Our proof starts with the observation that $O_{p^{\prime}}(G)$ is a $\mathcal{B}_{p^{-}}$-group and so it is contained in $\delta_{p^{\prime} p}(G)$. Let $T / O_{p^{\prime}}(G)=\delta_{p^{\prime} p}\left(G / O_{p^{\prime}}(G)\right)$. We shall prove that $T$ $\leqslant \delta_{p^{\prime} p}(G)$. From Lemma 1 and Theorem 6 we see that $\mu_{p}(G) / O_{p^{\prime}}(G)=\mu\left(G / O_{p^{\prime}}(G)\right)$ $\leqslant \mu_{p}\left(G / O_{p^{\prime}}(G)\right) \leqslant \delta_{p^{\prime} p}\left(G / O_{p^{\prime}}(G)\right)$ and hence $\mu_{p}(G) \leqslant T$. Thus the group $T / \mu_{p}(G)$ is 


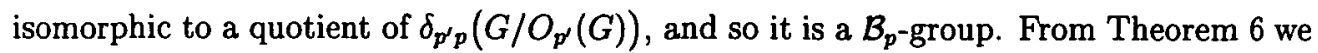
conclude that $T / \mu_{p}(G) \leqslant \delta_{p^{\prime} p}\left(G / \mu_{p}(G)\right)=\delta_{p^{\prime} p}(G) / \mu_{p}(G)$ and $T \leqslant \delta_{p^{\prime} p}(G)$.

In [4] we obtain a description of the Fitting subgroup of a group in terms of the locally nilpotent radical and the radicable part. Now we obtain the corresponding description of the $\mathcal{B}_{p}$-radical of a group. This result will be very useful in the characterisation of the $\mathcal{B}_{p}$-injectors in the next section.

LEMMA 2. Let $G$ be a group and let $p$ be a prime. Then $\delta_{p^{\prime} p}(G)=O_{p^{\prime} p}(G)$ $\cap C_{G}\left(\left(G^{0}\right)_{p}\right)$. Here $\left(G^{0}\right)_{p}$ denotes the Sylow p-subgroup of $G^{0}$.

Proof: Write $X=O_{p^{\prime} p}(G) \cap \mathrm{C}_{G}\left(\left(G^{0}\right)_{p}\right)$. Obviously $X$ is a normal $p$-nilpotent subgroup of $G$. Let $P$ be a Sylow $p$-subgroup of $X$. Since $G^{0}$ is Abelian we have that it is contained in $X$ and so $\left(X^{0}\right)_{p}=\left(G^{0}\right)_{p}$. Moreover $P^{0}=\left(X^{0}\right)_{p}$. We deduce that $P^{0}=\left(G^{0}\right)_{p}$ and $P^{0} \leqslant Z(P)$. Furthermore $P$ is locally nilpotent and it is a Chernikov group. We conclude that $P$ is nilpotent and so $X$ is a $\mathcal{B}_{p}$-group. Conversely, every $\mathcal{B}_{p^{-}}$ group is $p$-nilpotent and so $\delta_{p^{\prime} p}(G) \leqslant O_{p^{\prime} p}(G)$. Let us prove that $\delta_{p^{\prime} p}(G) \leqslant \mathrm{C}_{G}\left(\left(G^{0}\right)_{p}\right)$. Let $P$ be a Sylow $p$-subgroup of $\delta_{p^{\prime} p}(G)$. Then $P^{0} \leqslant \mathrm{Z}(P)$ by [6, (1.5.12)]. Since $G^{0} \leqslant \delta_{p^{\prime} p}(G)$ it follows that $G^{0}=\left(\delta_{p^{\prime} p}(G)\right)^{0}$. Then $\left(G^{0}\right)_{p} \leqslant \mathrm{Z}(P)$. On the other hand, $O_{p^{\prime}}\left(\delta_{p^{\prime} p}(G)\right)$ is the Sylow $p^{\prime}$-subgroup of $\delta_{p^{\prime} p}(G)$ and so $\delta_{p^{\prime} p}(G)=P O_{p^{\prime}}\left(\delta_{p^{\prime} p}(G)\right)$ by $[\mathbf{6}$, (3.1.6)]. Since $\left(G^{0}\right)_{p}$ centralises $O_{p^{\prime}}\left(\delta_{p^{\prime} p}(G)\right)$ we conclude that $\left(G^{0}\right)_{p}$ centralises $\delta_{p^{\prime} p}(G)$ and so $\delta_{p^{\prime} p}(G) \leqslant \mathrm{C}_{G}\left(\left(G^{0}\right)_{p}\right)$, which completes the proof.

\section{INJECTORS}

In infinite groups the definition of Fitting class is done in terms of the different generalisations of subnormality. In this paper we shall be concerned with Fitting classes defined using descendant subgroups. We say that a subclass $\mathcal{F}$ of $c \overline{\mathcal{L}}$ is a $c \overline{\mathcal{L}}$-Fitting class if it satisfies the following properties:

1. If $G \in \mathcal{F}$ and $H$ is a descendant subgroup of $G$, then $H \in \mathcal{F}$.

2. If $G=\left\langle H_{i}: i \in I\right\rangle \in c \overline{\mathcal{L}}$ and, for each $i \in I$ the subgroup $H_{i}$ is a descendant $\mathcal{F}$-subgroup of $G$, then $G \in \mathcal{F}$.

Applying Theorem $\mathrm{D}$, the subgroup generated by descendant $\mathcal{B}$-subgroups of a group is a $\mathcal{B}$-group. Therefore it is clear that $\mathcal{B}$ is a $c \overline{\mathcal{L}}$-Fitting class with the above definition. The corresponding injectors have been obtained in [4]. We prove in the sequel that $\mathcal{B}_{p}$ is also a Fitting class and study the $\mathcal{B}_{p}$-injectors. We begin with the local version of Theorem D.

THEOREM 7. Let $H$ be a descendant $\mathcal{B}_{p}$-subgroup of a group $G$. Then $H$ is contained in $\delta_{p^{\prime} p}(G)$.

Proof: Since $G$ is hyperfinite we apply [6, (7.2.11)] to conclude that $H$ is ascendant in $G$. By $[11,(1.31)], H \leqslant O_{p^{\prime} p}(G)$ because $H$ is $p$-nilpotent. If $H \leqslant \delta_{p^{\prime} p}\left(O_{p^{\prime} p}(G)\right)$ then 
$H \leqslant \delta_{p^{\prime} p}(G)$. Then there is no loss of generality in assuming that $G$ is $p$-nilpotent. From $[6,(3.1 .6)]$ it follows that $G=P O_{p^{\prime}}(G)$ for every Sylow $p$-subgroup $P$ of $G$. The task is now to show that if $P$ is a Sylow $p$-subgroup of $G$ then $F(P)$ is a Sylow $p$-subgroup of $\delta_{p^{\prime} p}(G)$. We have that $F(P)$ is nilpotent by Theorem A. It follows that $F(P) O_{p^{\prime}}(G)$ is in the class $\mathcal{B}_{p}$ and so $F(P) O_{p^{\prime}}(G)$ is contained in $\delta_{p^{\prime} p}(G)$ because it is normal in $P O_{p^{\prime}}(G)=G$. On the other hand, $P \cap \delta_{p^{\prime} p}(G)$ is nilpotent. Consequently, we have that $F(P)=P \cap \delta_{p^{\prime} p}(G)$ and thus $\delta_{p^{\prime} p}(G)=F(P) O_{p^{\prime}}(G)$. Hence $F(P)$ is a Sylow $p$ subgroup of $\delta_{p^{\prime} p}(G)$. Since $H$ is $p$-nilpotent, it follows that $H=P_{H} O_{p^{\prime}}(H)$ where $P_{H}$ is a Sylow $p$-subgroup of $H$. Moreover, there exists a Sylow $p$-subgroup $P$ of $G$ such that $P_{H}=P \cap H$. Consequently $P_{H}$ is a nilpotent descendant subgroup of $P$ and hence, applying Theorem D, $P_{H} \leqslant F(P)$. Moreover $O_{p^{\prime}}(H) \leqslant O_{p^{\prime}}(G)$ by $[11,(1.31)]$. We conclude that $H=P_{H} O_{p^{\prime}}(H) \leqslant F(P) O_{p^{\prime}}(G)=\delta_{p^{\prime} p}(G)$.

Corollary 5. Let $H$ be a descendant subgroup of a group $G$. Then $\delta_{p^{\prime} p}(G)$ $\cap H=\delta_{p^{\prime} p}(H)$.

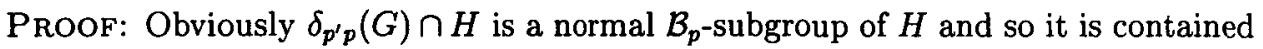
in $\delta_{p^{\prime} p}(H)$. Conversely, since $H$ is descendant in $G$ it follows that $\delta_{p^{\prime} p}(H)$ is a descendant $\mathcal{B}_{p}$-subgroup of $G$ and hence, by Theorem 7 , it is contained in $\delta_{p^{\prime} p}(G)$.

As a consequence of Theorem 7 , the subgroup generated by descendant $\mathcal{B}_{p}$-subgroups of a group is a $\mathcal{B}_{p}$-group. We conclude that $\mathcal{B}_{p}$ is a $c \overline{\mathcal{L}}$-Fitting class. Note that this is not true if we replace, in the definition of a Fitting class, descendant subgroups by serial subgroups (which are in fact ascendant in a $c \overline{\mathcal{L}}$-group). For instance, the locally dihedral 2 -group is an example of a join of serial $\mathcal{B}_{2}$-subgroups which is not a $\mathcal{B}_{2}$-group. Moreover, this group has ascendant $\mathcal{B}_{2}$-subgroups which are not contained in the $\mathcal{B}_{2}$-radical.

Let $\mathcal{F}$ be a subclass of $c \overline{\mathcal{L}}$ and $G \in c \overline{\mathcal{L}}$. An $\mathcal{F}$-injector of $G$ is a subgroup $V$ of $G$ such that for all descendant subgroups $H$ of $G$ we have that $V \cap H$ is $\mathcal{F}$-maximal in $H$. We denote the set of all $\mathcal{F}$-injectors of the group $G$ by $\operatorname{Inj}_{\mathcal{F}}(G)$.

The following result describes the injectors for the Fitting class of all $p$-nilpotent groups.

ThEOREM 8 . Let $G$ be a group. Then

$$
\operatorname{Inj}_{\mathfrak{G}_{p^{\prime}} \mathfrak{G}_{p}}(G)=\left\{P O_{p^{\prime}}(G): P \text { is a Sylow } p \text {-subgroup of } G\right\} .
$$

Therefore the p-nilpotent injectors of $G$ are exactly the maximal p-nilpotent subgroups of $G$ containing $O_{p^{\prime} p}(G)$ and they form a conjugacy class of subgroups of $G$.

Proof: Let $P$ be a Sylow $p$-subgroup of $G$. We prove that $P O_{p^{\prime}}(G)$ is a $p$-nilpotent injector of $G$. Let us first show that $P O_{p^{\prime}}(G)$ is a maximal $p$-nilpotent subgroup. Obviously $P O_{p^{\prime}}(G)$ is $p$-nilpotent. Let $W$ be a $p$-nilpotent subgroup of $G$ such that $P O_{p^{\prime}}(G)$ $\leqslant W$. It follows that $P$ is a Sylow $p$-subgroup of $W$ and so $W=P O_{p^{\prime}}(W)$. Therefore, it remains to prove that $O_{p^{\prime}}(W)=O_{p^{\prime}}(G)$. Since $O_{p^{\prime}}(W) / O_{p^{\prime}}(G)=O_{p^{\prime}}\left(W / O_{p^{\prime}}(G)\right)$, 
there is no loss of generality in assuming that $O_{p^{\prime}}(G)=1$ and proving that $O_{p^{\prime}}(W)=1$. According to [6, (2.5.13)], $G$ is a Chernikov group, that is $G=G^{0} A$ where $A$ is a finite subgroup of $G$. We can certainly assume that $G^{0} \neq 1$, since otherwise $G$ is finite and then the result is true (see [8]). Since $O_{p^{\prime}}(G)=1$, we have that $G^{0}$ is a $p$-group. Therefore $O_{p^{\prime}}(W)$ is finite. Suppose, contrary to our claim, that $O_{p^{\prime}}(W) \neq 1$. Write $A_{p}=O_{p}(G) \cap A$. Then $G^{0} A_{p}=O_{p}(G)$. Since $O_{p}(G) \leqslant P \leqslant W$ it follows that $A_{p} \leqslant W$ and hence $A_{p}$ normalises $O_{p^{\prime}}(W)$. By $([7,($ A.12.5)]), we have that $O_{p^{\prime}}(W)=\left[O_{p^{\prime}}(W), A_{p}\right] \mathrm{C}_{O_{p^{\prime}}(W)}\left(A_{p}\right)$. Moreover, $\left[O_{p^{\prime}}(W), A_{p}\right] \leqslant O_{p}(G) \cap O_{p^{\prime}}(W)=1$. We deduce that $O_{p^{\prime}}(W)=\mathrm{C}_{O_{p^{\prime}}(W)}\left(A_{p}\right)$, that is, $O_{p^{\prime}}(W) \leqslant \mathrm{C}_{G}\left(A_{p}\right)$. On the other hand, $G^{0}=\left[G^{0}, O_{p^{\prime}}(W)\right] \mathrm{C}_{G^{0}}\left(O_{p^{\prime}}(W)\right)$ by $[11,(3.29 .1)]$. Since $G^{0} \leqslant P \leqslant W$ we have that $G^{0}$ normalises $O_{p^{\prime}}(W)$ and so $\left[G^{0}, O_{p^{\prime}}(W)\right] \leqslant G^{0} \cap O_{p^{\prime}}(W)=1$. Consequently $G^{0}=\mathrm{C}_{G^{0}}\left(O_{p^{\prime}}(W)\right)$, that is $O_{p^{\prime}}(W) \leqslant \mathrm{C}_{G}\left(G^{0}\right)$. Since $G^{0} A_{p}=O_{p}(G)$ we conclude that $O_{p^{\prime}}(W) \leqslant \mathrm{C}_{G}\left(O_{p}(G)\right) \leqslant O_{p}(G) \neq 1$, a contradiction. Therefore $O_{p^{\prime}}(W)=1$ and thus we have proved that $P O_{p^{\prime}}(G)$ is a maximal p-nilpotent subgroup of $G$. We are now in position to show that if $H$ is a descendant subgroup of $G$, then $P O_{p^{\prime}}(G) \cap H$ is a maximal $p$-nilpotent subgroup of $H$ by the above argument. Since $P \cap H$ is a Sylow $p$-subgroup of $H$ by [5, (2.7)], we have that $(P \cap H) O_{p^{\prime}}(H)$ is a maximal $p$-nilpotent subgroup of $H$. Moreover, $O_{p^{\prime}}(H) \leqslant O_{p^{\prime}}(G)$. Therefore $(P \cap H) O_{p^{\prime}}(H)=\left(O_{p^{\prime}}(H) P\right) \cap H \leqslant\left(O_{p^{\prime}}(G) P\right) \cap H$ and hence $(P \cap H) O_{p^{\prime}}(H)=\left(O_{p^{\prime}}(G) P\right) \cap H$ because $\left(O_{p^{\prime}}(G) P\right) \cap H$ is $p$-nilpotent. We conclude that $\left(O_{p^{\prime}}(G) P\right) \cap H$ is $\mathfrak{S}_{p^{\prime}} \mathfrak{S}_{p^{\prime}}$-maximal in $H$ and so we have that $P O_{p^{\prime}}(G)$ is a $p$-nilpotent injector of $G$. We shall be done if we show that every $p$-nilpotent injector appears in that way. Let $I$ be a $p$-nilpotent injector of $G$. In particular we can write $I=O_{p^{\prime}}(I) I_{p}$ where $I_{p}$ is a Sylow $p$-subgroup of $I$. We show that $O_{p^{\prime}}(I)=O_{p^{\prime}}(G)$. Clearly, since $I$ is a $p$-nilpotent injector of $G$, it follows that $O_{p^{\prime} p}(G) \leqslant I$ and thus $O_{p^{\prime}}(G) \leqslant O_{p^{\prime}}(I)$. Because $O_{p^{\prime}}(I) / O_{p^{\prime}}(G)=O_{p^{\prime}}\left(I / O_{p^{\prime}}(G)\right)$, there is no loss of generality in assuming that $O_{p^{\prime}}(G)=1$. Arguing as above we deduce that $O_{p^{\prime}}(I)=1$. Therefore $I=O_{p^{\prime}}(I) I_{p}$ is contained in the $p$-nilpotent subgroup $O_{p^{\prime}}(G) P$ where $P$ is a Sylow $p$-subgroup of $G$. We conclude, by maximality of $I$, that $I=O_{p^{\prime}}(G) P$, which is our claim.

Furthermore, by the same method as above, it can be proved that every maximal $p$ nilpotent subgroup $V$ of $G$ containing $O_{p^{\prime} p}(G)$ can be described as $V=P O_{p^{\prime}}(G)$ where $P$ is a Sylow $p$-subgroup of $G$, and consequently $V$ is a $p$-nilpotent injector of $G$. Therefore the maximal $p$-nilpotent subgroups of a group containing the $p$-nilpotent radical are precisely the $p$-nilpotent injectors.

Note that using the same arguments to those used in the proof of Theorem 8 we have that if $V$ is a $p$-nilpotent injector of a group $G$ and $H$ is an ascendant subgroup of $G$, then $V \cap H$ is a $p$-nilpotent injector of $G$.

We now proceed to show that the $\mathcal{B}_{p}$-injectors of a group can be described in terms of the $p$-nilpotent injectors of one of its subgroups. We require a preliminary result. It deals with the situation in which we take a product of a $\mathcal{B}_{p}$-subgroup of a descendant 
subgroup with the $\mathcal{B}_{p}$-radical.

LemMa 3. Let $G$ be a group and let $W$ be a $\mathcal{B}_{p}$-subgroup of $G$. Suppose that $H$ is a descendant subgroup of $G$ and $\delta_{p^{\prime} p}(H) \leqslant W \leqslant H$. Then $W \delta_{p^{\prime} p}(G)$ is a $\mathcal{B}_{p}$-subgroup of $G$.

Proof: By Corollary 5 we have that $\delta_{p^{\prime} p}(G) \cap H=\delta_{p^{\prime} p}(H)$. Moreover, applying Theorem 7 , the join of descendant $\mathcal{B}_{p}$-subgroups is a $\mathcal{B}_{p}$-group. Using these facts, the result follows by the same method as in $[6,(7.3 .5)]$.

Theorem 9. Let $G$ be a group. Then the $\mathcal{B}_{p}$-injectors of $G$ are exactly the $p$ nilpotent injectors of $C=C_{G}\left(\left(G^{0}\right)_{p}\right)$. In particular the $\mathcal{B}_{p}$-injectors of $G$ are conjugate in $G$.

Proof: Denote $C=\mathrm{C}_{G}\left(\left(G^{0}\right)_{p}\right)$ and let $V$ be a $p$-nilpotent injector of $C$. We show that $V \in \operatorname{Inj}_{\mathcal{B}_{p}}(G)$. According to Lemma $2, \delta_{p^{\prime} p}(G)=O_{p^{\prime} p}(G) \cap C=O_{p^{\prime} p}(C)$. Of course, $O_{p^{\prime} p}(C) \leqslant V$ because $V$ is a $p$-nilpotent injector of $C$. It follows that $\delta_{p^{\prime} p}(G) \leqslant V$. Let $H$ be a descendant subgroup of $G$ and suppose that $W$ is a $\mathcal{B}_{p}$-subgroup of $H$ such that $V \cap H \leqslant W$. From Corollary 5, we have that $\delta_{p^{\prime} p}(H)=\delta_{p^{\prime} p}(G) \cap H \leqslant V \cap H \leqslant W$ $\leqslant H$. This clearly forces that $W \delta_{p^{\prime} p}(G)$ is a $\mathcal{B}_{p}$-group by Lemma 3 . Since $G^{0} \leqslant \delta_{p^{\prime} p}(G)$ we have that $\left(G^{0}\right)_{p}$ is contained in a Sylow $p$-subgroup $P$ of $W \delta_{p^{\prime} p}(G)$. Consequently

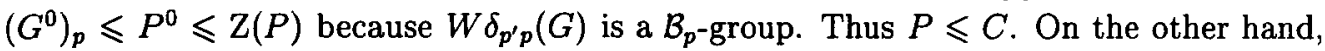
$O_{p^{\prime}}\left(W \delta_{p^{\prime} p}(G)\right) \leqslant C$. Since $W \delta_{p^{\prime} p}(G)=O_{p^{\prime}}\left(W \delta_{p^{\prime} p}(G)\right) P$ we conclude that $W \delta_{p^{\prime} p}(G)$ $\leqslant C$. In particular, $W$ is contained in $C$. On the other hand, $V \cap H=V \cap(H \cap C)$ is a maximal $p$-nilpotent subgroup of $H \cap C$ because $V$ is a $p$-nilpotent injector of $C$. Since $V \cap H \leqslant W \leqslant H \cap C$ it follows that $V \cap H=W$. Therefore $V \cap H$ is a maximal $\mathcal{B}_{p^{-}}$ subgroup of $H$, and we have proved that $V \in \operatorname{Inj}_{\mathcal{B}_{p}}(G)$. Conversely, suppose that $V$ is a $\mathcal{B}_{p}$-injector of $G$. Let us first observe that $V$ is contained in $C$. Clearly $G^{0} \leqslant \delta_{p^{\prime} p}(G) \leqslant V$. It follows that $\left(G^{0}\right)_{p}$ is normal in $V$, and consequently $O_{p^{\prime}}(V) \leqslant \mathrm{C}_{G}\left(\left(G^{0}\right)_{p}\right)$. Moreover $\left(G^{0}\right)_{p} \leqslant V_{p}$ where $V_{p}$ is a Sylow $p$-subgroup of $V$ and $V_{p}^{0} \leqslant \mathrm{Z}\left(V_{p}\right)$. Therefore $V_{p} \leqslant C$. Since $V=O_{p^{\prime}}(V) V_{p}$ we conclude that $V \leqslant C$. We may now prove that $V$ is a $p$-nilpotent injector of $C$. Let $D$ be a descendant subgroup of $C$ and suppose that $W$ is a $p$-nilpotent subgroup of $D$ such that $V \cap D \leqslant W$. It is easy to check that $W$ is a $\mathcal{B}_{p}$-group. Since $D$ is also descendant in $G$ we deduce that $V \cap D=W$ because $V$ is a $\mathcal{B}_{p}$-injector of $G$. We conclude that $V$ is a $p$-nilpotent injector of $C$ as required.

Finally, we can show that, in fact, the maximal $\mathcal{B}_{p}$-subgroups of a group containing the $\mathcal{B}_{p}$-radical are precisely the $\mathcal{B}_{p}$-injectors.

THEOREM 10. The maximal $\mathcal{B}_{p}$-subgroups of a group $G$ which contain the $\mathcal{B}_{p^{-}}$ radical of $G$ are precisely the $\mathcal{B}_{p}$-injectors of $G$.

Proof: Obviously, every $\mathcal{B}_{p}$-injector of $G$ is a maximal $\mathcal{B}_{p}$-subgroup of $G$ and contains the $\mathcal{B}_{p}$-radical of $G$. Conversely, let $V$ be a maximal $\mathcal{B}_{p}$-subgroup of $G$ such that $\delta_{p^{\prime} p}(G) \leqslant V$. We wish to show that $V \in \operatorname{Inj}_{\mathcal{B}_{p}}(G)$. By Theorem 9 , we are reduced to 
proving that $V$ is a $p$-nilpotent injector of $\mathrm{C}_{G}\left(\left(G^{0}\right)_{p}\right)$. Write $C=\mathrm{C}_{G}\left(\left(G^{0}\right)_{p}\right)$. Arguing as in the proof of Theorem 9 , it is easily seen that $V \leqslant C$. We proceed to show that $V$ is a maximal $p$-nilpotent subgroup of $C$. Clearly $V$ is $p$-nilpotent because it is a $\mathcal{B}_{p}$-group. Suppose that $W$ is a $p$-nilpotent subgroup of $C$ such that $V \leqslant W$. It is clear that $W$ is a $\mathcal{B}_{p}$-group. Therefore, since $V$ is a maximal $\mathcal{B}_{p}$-subgroup of $G$, it follows that $W=V$ and hence $V$ is a maximal $p$-nilpotent subgroup of $C$. Furthermore, applying Lemma 2, $\delta_{p^{\prime} p}(G)=O_{p^{\prime} p}(G) \cap C=O_{p^{\prime} p}(C)$. Therefore $V$ is a maximal $p$-nilpotent subgroup of $C$ containing $O_{p^{\prime} p}(C)$. As a consequence of Theorem 8 , we deduce that $V$ is a $p$-nilpotent injector of $C$, which is the desired conclusion.

\section{REFERENCES}

[1] A. Ballester-Bolinches and S. Camp-Mora, 'A Gaschütz-Lubeseder type theorem in a class of locally finite groups', J. Algebra 221 (1999), 562-569.

[2] A. Ballester-Bolinches and S. Camp-Mora, 'A Bryce-Cossey type theorem in a class of locally finite groups', Bull. Austral. Math. Soc. 63 (2001), 459-466.

[3] A. Ballester-Bolinches and T. Pedraza, 'On a class of generalized nilpotent groups', $J$. Algebra 248 (2002), 219-229.

[4] A. Ballester-Bolinches and T. Pedraza, 'The fitting subgroup and some injectors of radical locally finite groups with min-p for all $p$ ', Comm. Algebra 31 (2003), 483-492.

[5] M.R. Dixon, 'Certain fitting classes of groups with min- $p$ for all $p$ ', Arch. Math. (Basel) 54 (1990), 521-532.

[6] M.R. Dixon, Sylow theory, formations and fitting classes in locally finite groups, Series in Algebra 2 (World Scientific, River Edge, N.J., 1994).

[7] K. Doerk and T. O. Hawkes, Finite soluble groups, de Gruyter Expositions in Mathematics 4 (Walter De Gruyter, Berlin, 1992).

[8] M.J. Iranzo and M. Torres, 'The $p^{*} p$-injectors of a finite group', Rend. Sem. Mat. Univ. Padova 82 (1989), 233-237.

[9] J. Lafuente, 'Chief factors of finite groups with order a multiple of $p$ ', Comm. Algebra 16 (1988), 1563-1580.

[10] D.J.S. Robinson, $A$ course in the theory of groups, Graduate Texts in Mathematics 80 (Springer-Verlag, New York, Berlin, 1982).

[11] D.J.S. Robinson, Finiteness conditions and generalized soluble groups, Volumes 1 and 2 (Springer-Verlag, Berlin, 1972).

[12] M.J. Tomkinson, 'A frattini-like subgroup', Math. Proc. Cambridge Philos. Soc. 77 (1975), 247-257.

[13] M.J. Tomkinson, 'Finiteness conditions and a frattini-like subgroup', Rend. Circ. Mat. Palermo 23 (1990), 321-335.

[14] M.J. Tomkinson, 'Schunck classes and projectors in a class of locally finite groups', Proc. Edinburgh Math. Soc. 38 (1995), 511-522. 
Departament d'Àlgebra

Universitat de València

Dr. Moliner 50

46100 Burjassot

València

Spain

e-mail: Adolfo.Ballester@uv.es
Departamento de Matemática Aplicada Universidad Politécnica de Valencia Escuela Politécnica Superior de Alcoy 3801 Alcoy

Alicante

Spain

e-mail: tapedraz@mat.upv.es 